\title{
Comunicação \\ Ação do lodo de curtume no controle de Meloidogyne spp. e na nodulação em soja
}

Fabio Fernando de Araujo ${ }^{1}$, Geisa de Melo Gentil ${ }^{2}$

\section{RESUMO}

O objetivo deste trabalho foi avaliar doses de lodo de curtume incorporadas ao solo sobre a nodulação e o controle de Meloidogyne spp. em soja. Foram empregadas doses crescentes (0; 5,0; 10,0; 20; 40,0; e $80 \mathrm{Mg} \mathrm{ha}^{-1}$ ) de lodo no solo. O solo de cada vaso foi infestado com 5.000 ovos de Meloidogyne spp após a semeadura da soja. As plantas foram conduzidas durante 70 dias em casa de vegetação e após esse período foram coletadas para avaliação da nodulação, massa de matéria seca da parte aérea e reprodução dos nematóides nas raízes. O aumento da dose de lodo de curtume aplicada ao solo reduziu de forma quadrática a reprodução do nematóide nas raízes de soja. Entretanto, o aumento da concentração de lodo no solo reduziu exponencialmente a nodulação da cultura e não proporcionou benefícios ao crescimento da planta.

Palavras-chave: Resíduos, reciclagem, nematóide.

\section{ABSTRACT}

\section{Tannery sludge in the control Meloidogyne spp. and nodulation in soybean}

The objective of this study was to evaluate rates of tannery sludge on the nodulation and the control of nematodes in soybean. Increasing rates of sludge $\left(0 ; 5.0 ; 10.0 ; 20.0 ; 40.0 ;\right.$ and $\left.80.0 \mathrm{Mg} \mathrm{ha}^{-1}\right)$ were applied into the soil. Soil was infested with 5,000 eggs of Meloidogyne spp. after soybean sowing. Seventy days later, in a greenhouse, nodulation, dry mass of the shoots and nematode reproduction in the roots were evaluated. The application of tannery sludge in the soil in higher doses reduced nematode reproduction in the roots of soybean. However, the increase in sludge concentration in the soil reduced nodulation and showed no benefit to plant growth.

Key words: Nematode, residue, recycling.

Recebido para publicação em novembro de 2008 e aprovado em julho de 2010

${ }^{1}$ Engenheiro Agrônomo, Doutor. Curso de Agronomia, Universidade do Oeste Paulista, Faculdade de Ciências Agrárias, Agronomia, Rodovia Raposo Tavares km 572, Limoeiro, 19050-900, Presidente Prudente, SP, Brasil. fabio@unoeste.br

${ }^{2}$ Acadêmica do curso de Agronomia, Universidade do Oeste Paulista, Faculdade de Ciências Agrárias, Agronomia. Rodovia Raposo Tavares km 572, Limoeiro, 19050-900, Presidente Prudente, SP, Brasil. 


\section{INTRODUÇÃO}

A expansão da cultura da soja no Brasil tem sido crescente, e dentre os principais fatores que limitam o aumento de sua produtividade estão as doenças (Soares et al., 2004). Entre as principais doenças do solo destacam-se as causadas por nematóides formadores de galhas nas raízes, pertencentes ao gênero Meloidogyne (Lordello, 1988). Esse gênero compreende grande número de espécies; entretanto, $M$. incognita, $M$. arenaria e $M$. javanica são as mais encontradas parasitando as raízes e causando redução no crescimento da soja cultivada nos solos brasileiros (Almeida et al., 1997).

O controle de nematóides em culturas anuais, como a soja, deve ser planejado de forma a integrar várias medidas de manejo, envolvendo o uso de diferentes princípios de controle de doenças em plantas (Kerry, 1987). Dentro das estratégias de controle desta doença tem sido priorizada a redução da comunidade do parasita no solo, destacandose métodos culturais, como a incorporação de resíduos orgânicos, que irá proporcionar aumento na biodiversidade da microfauna do solo (Araujo \& Bettiol, 2005).

O lodo de curtume tem sua disposição no solo normatizada por órgãos ambientais (Cetesb, 1999). Dessa forma, com o alto custo dos fertilizantes comerciais e a dificuldade de disposição final de resíduos industriais, a utilização do lodo de curtume na agricultura, seguindo critérios técnicos e com monitoramento periódico, torna-se alternativa viável, tanto do ponto de vista econômico quanto do ambiental. Devido à ocorrência da fixação biológica de nitrogênio em leguminosas, a utilização de resíduos orgânicos nessas culturas deve ser também avaliada quanto ao impacto que essa prática possa causar no processo simbiótico desenvolvido entre o rizóbio e a planta hospedeira (Angle et al., 1993).

Em razão do potencial da incorporação de resíduos orgânicos ao solo para o estímulo do crescimento de plantas e a supressividade às doenças do solo, o objetivo deste trabalho foi avaliar o efeito de doses de lodo de curtume incorporadas ao solo sobre o crescimento, a nodulação e o controle do nematóide de galhas em soja.

\section{MATERIAL E MÉTODOS}

$\mathrm{O}$ estudo foi realizado durante os meses de março a junho de 2008. O solo utilizado, classificado como Latossolo Vermelho-Amarelo (Embrapa, 1999), apresentava textura arenosa. A análise química do solo foi realizada segundo Van Raij et al. (1983), apresentando os seguintes resultados: $\mathrm{pH}\left(\mathrm{CaCl}_{2} 1 \mathrm{~mol} \mathrm{~L}^{-1}\right) 6,0 ; 6 \mathrm{~g} \mathrm{dm}^{-3}$ de M.O.; $38 \mathrm{mg} \mathrm{dm}^{-3}$ de $\mathrm{P}_{\text {resina }}$; 3,6 $\mathrm{mmol}_{\mathrm{c}} \mathrm{dm}^{-3} \mathrm{de} \mathrm{K}$; $24 \mathrm{mmol}_{\mathrm{c}} \mathrm{dm}^{-3} \mathrm{de} \mathrm{Ca} ; 8 \mathrm{mmol}_{c} \mathrm{dm}^{-3} \mathrm{de} \mathrm{Mg} ; 51 \mathrm{mmol}_{\mathrm{c}} \mathrm{dm}^{-3} \mathrm{de}$ CTC; e $70 \%$ de saturação de bases.

Rev. Ceres, Viçosa, v. 57, n.5, p. 629-632, set/out, 2010
O lodo de curtume utilizado no experimento foi proveniente da etapa da ribeira obtido no Curtume Vitapelli, Presidente Prudente, SP. A análise química (Malavolta et al. 1997) do resíduo (base seca) indicou as seguintes concentrações de nutrientes $\left(\mathrm{g} \mathrm{kg}^{1}\right)$ : 29,1 de N; 6,1 de P; 0,6 de K; 128,5 de Ca; 10,8 de Mg; e 13,0 de S.

O experimento foi conduzido em vasos com capacidade de $5 \mathrm{~kg}$ de solo. $O$ delineamento experimental utilizado foi de blocos inteiramente casualizados, com quatro repetições. O lodo do curtume foi misturado ao solo antes do enchimento dos vasos e o do plantio da soja. A quantidade de lodo aplicada ao solo foi estabelecida considerando-se o fornecimento de $\mathrm{N}$ pelo resíduo, com taxa de mineralização de 30 \% (Cetesb, 1999). Os tratamentos com fornecimento de $0,50,100,200,400$ e $800 \mathrm{~kg}$ de $\mathrm{N} \mathrm{ha}^{-1}$ foram efetivados com a incorporação das seguintes doses de lodo (base seca) por hectare: $0 \mathrm{Mg} \mathrm{ha}^{-1}$ (ausência de lodo); 5,0 $\mathrm{Mg} \mathrm{ha}^{-1}$ (12,5 g de lodo por vaso); 10,0 Mg ha-1 (25 g de lodo por vaso); 20,0 $\mathrm{Mg} \mathrm{ha}^{-1}$ (50 g de lodo por vaso); 40,0 $\mathrm{Mg} \mathrm{ha}^{-1}$ (100 g de lodo por vaso); e $80,0 \mathrm{Mg}$ ha $^{-1}$ (200 g de lodo por vaso). Após o enchimento dos vasos com o solo de cada tratamento foi realizada a semeadura de cinco sementes de soja por vaso. Essas foram previamente inoculadas com Bradyrhizobium spp. com a utilização de inoculante turfoso comercial (Turfal Ltda.), contendo 1,0 $10^{9}$ bactéria por grama do produto, empregando-se a dose de $500 \mathrm{~g}$ por $50 \mathrm{~kg}$ de semente. O cultivar de soja utilizado no experimento foi o BRS 245.

Após a semeadura, o solo de cada vaso foi infestado com 5.000 ovos de Meloidogyne spp., obtidos de raízes de soja com sintomas de galhas e extraídos de acordo com a técnica de Hussey \& Barker (1973). As plantas foram conduzidas durante 77 dias e, após esse período, foram coletadas cuidadosamente, separando-se a parte aérea da raiz. No laboratório, os nódulos foram separados da raiz e colocados para secar, juntamente com a parte aérea, em estufa $\left(70^{\circ} \mathrm{C}\right)$ até atingirem peso constante. Para a avaliação da infestação de nematóides, as raízes frescas foram pesadas previamente e trituradas em liquidificador e em seguida quantificados os ovos e juvenis de nematóides (Hussey \& Barker, 1973). O fator de reprodução foi calculado com base na relação entre o número de ovos de nematoide encontrado na raiz e o número de ovos inoculados no solo, conforme descrito por Araújo \& Bettiol (2005). Os dados foram submetidos à análise de regressão, utilizando-se o programa Sisvar.

\section{RESULTADOS E DISCUSSÃO}

Na Figura 1-a observa-se que não houve ajuste de função matemática relacionando doses de lodo de curtume aplicadas ao solo e produção de massa de matéria seca de soja. A incorporação de diferentes doses de lodo ao solo não proporcionou incrementos na massa de matéria seca 

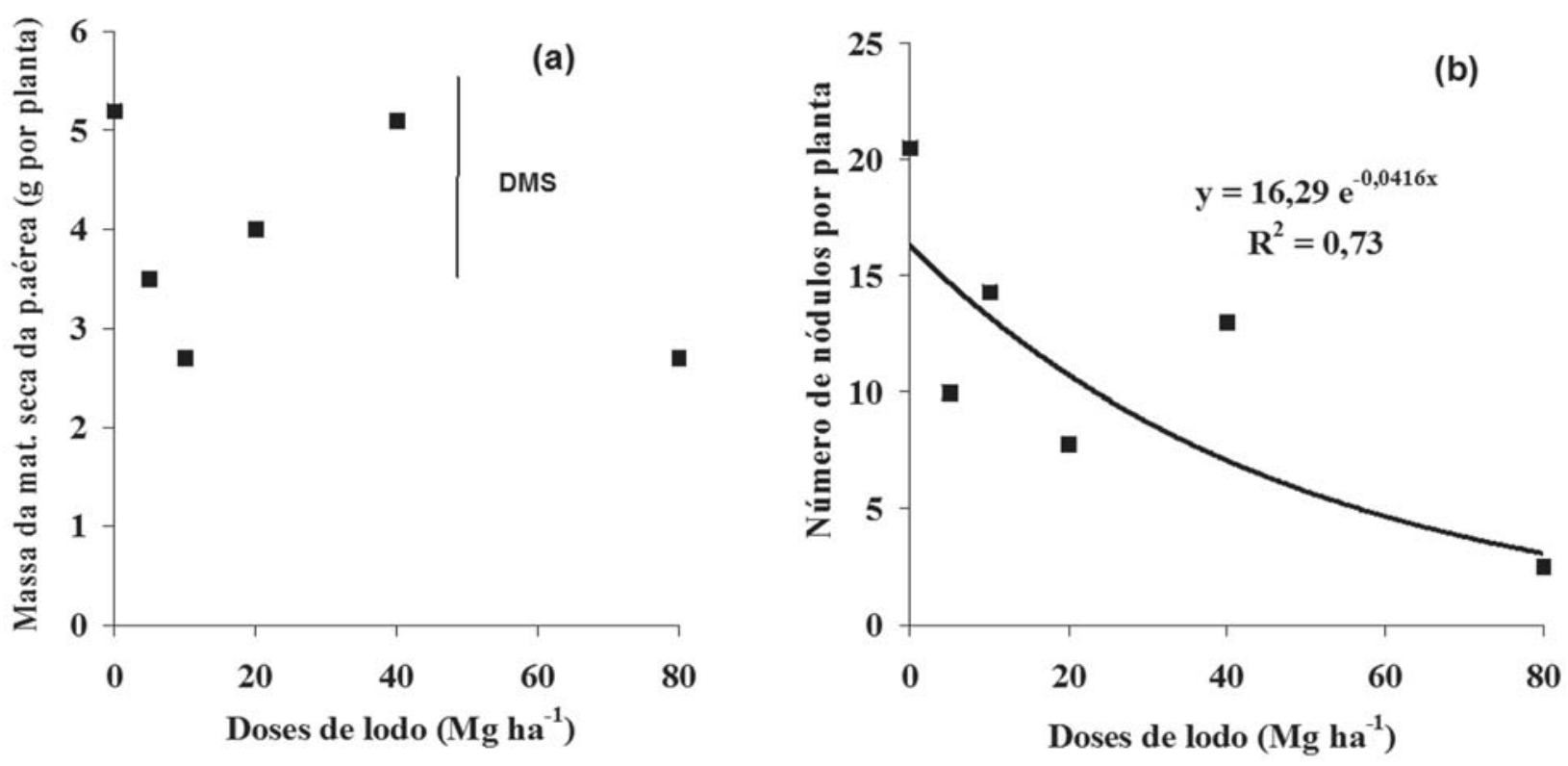

Figura 1. Massa de matéria seca da parte aérea (a) e número de nódulos (b) em plantas de soja em razão de doses de lodo de curtume incorporado ao solo.

das plantas em comparação à testemunha. A análise de regressão para a variável número de nódulos por planta em função da dose de lodo de curtume apresentou o modelo exponencial como de melhor ajuste, revelando que já nas doses menores de lodo incorporadas ao solo ocorreu efeito negativo na nodulação da soja (Figura 1-b).

Em trabalho com incorporação de lodo de curtume em cultivo de soja foi encontrado que a dose que proporcionou o melhor crescimento da cultura foi de 21 toneladas por hectare (Ferreira et al., 2003). Em outro trabalho, que testou a aplicação de doses correspondentes a 11,2 e 22,4 $\mathrm{Mg} \mathrm{ha}^{-1}$ de lodo de esgoto verificou-se decréscimo de nodulação na soja quando se aplicou a maior dose (Vieira, 2004). Neste trabalho com lodo de curtume as doses acima de 5,0 $\mathrm{Mg} \mathrm{ha}^{-1}$ ocasionaram perdas na nodulação da planta e não incrementaram o crescimento da soja. A causa do efeito negativo sobre a nodulação não foi identificada, mas pode estar relacionada, entre outras coisas, com o acúmulo de metais ou sais solúveis no solo. Bradyrhizobium japonicum apresenta alta tolerância intrínseca na presença de metais pesados e a possibilidade de que metais pesados presentes no lodo afetem a população no solo, em curto prazo, é remota (Angle et al., 1992). Sabe-se também que a fitotoxicidade do lodo de curtume não é devida apenas à presença de metais pesados, mas também a concentrações de sais, que aumentam fortemente a condutividade elétrica do solo (Ferreira \& Castro, 1995).

O modelo quadrático obtido no ajuste do efeito das doses de lodo de curtume sobre a reprodução dos nematóides apresentou correlação significativa, mostrando tendência de redução da reprodução do parasita quando se aumenta a incorporação do lodo ao solo (Figura 2).
Araújo \& Bettiol (2005), utilizando lodo de esgoto para controle de nematóides no solo, encontraram que o fator de reprodução de $M$. javanica foi reduzido nos tratamentos que o receberam na concentração de $20 \mathrm{Mg} \mathrm{ha}^{-1}$.

O efeito da adição do lodo ao solo na redução da reprodução do nematoide de galhas ( $M$. javanica) pode ter sido provocado pela presença de antagonistas no solo, que, estimulados pela presença de matéria orgânica, tenham interferido na eclosão e orientação das larvas, além de parasitarem os ovos do nematoide (Kerry, 1987). Sabese que o ovo pode ser considerado o estágio mais vulnerável no ciclo de vida de Meloidogyne spp. (Viaene \& Abawi, 1998). Reforçando essa afirmação, Chen et al.

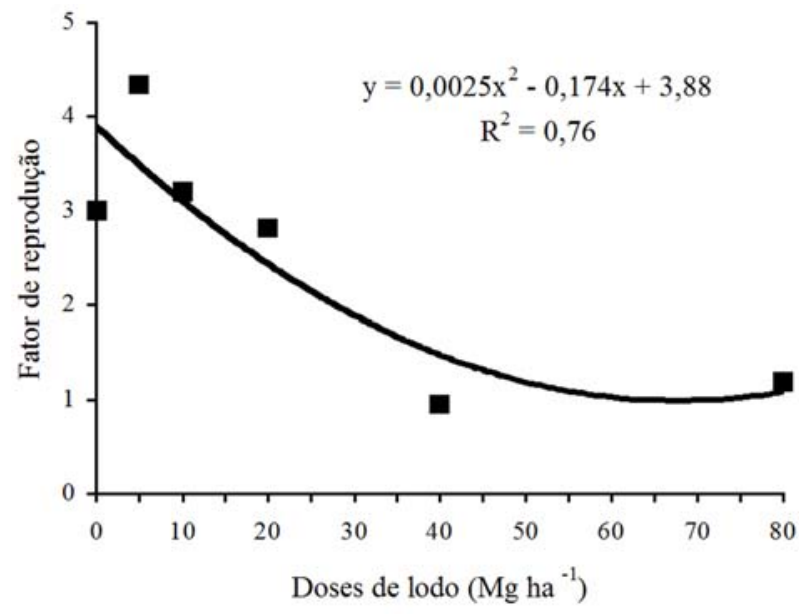

Figura 2. Fator de reprodução de nematóides de galhas em soja aos 80 dias de idade em razão da incorporação de doses de lodo de curtume ao solo. 
(2000) citaram que a aplicação de composto de cervejaria reduziu a produção de ovos de M. hapla.

Alguns trabalhos relataram que resíduos orgânicos, quando introduzidos ao solo, sofrem intensa mineralização microbiana com formação do íon $\mathrm{NH}_{4}^{+}$, o que pode proporcionar efeito nematicida (Pereira et al., 1996; RodríguezKábana, 1986). Foi encontrado que em lodos de tratamento biológico o $\mathrm{NH}_{4}^{+}$foi a forma predominante de $\mathrm{N}$ mineral no sistema lodo-solo nas primeiras semanas após a aplicação, em seguida essa forma diminuiu, havendo aumento correspondente de $\mathrm{NO}_{3}^{+}$e $\mathrm{NO}_{2}^{-}$(Boeira et al., 2002).

\section{CONCLUSÃO}

O aumento da dose de lodo de curtume aplicada ao solo reduziu de forma quadrática a reprodução do nematoide nas raízes de soja. Entretanto, essa prática afeta consideravelmente a nodulação da cultura e não proporciona benefícios ao crescimento da planta.

\section{REFERÊNCIAS}

Angle JS, Mcgrath SP, Chaudri AM, Chaney RL \& Giller KE (1993) Inoculation effects on legumes grown in soil previously treated with sewage sludge. Soil Biology and Biochemistry, 25:575-580.

Angle JS, Madariaga GM \& Heger EA (1992) Sewage sludge effects on growth and nitrogen fixation of soybean. Agriculture, Ecosystems and Environment, 41:231-239.

Almeida AMR, Ferreira LP, Yorinori JT, Silva JFV \& Henning AA (1997) Doenças da soja. In: Kimati H, Amorim L, Bergamin Filho A, Camargo LEA \& Rezende JAM (Eds.) Manual de Fitopatologia. São Paulo, Editora Agronômica Ceres. p. 642664.

Araújo FF \& Bettiol W (2005) Supressividade dos nematóides Meloidogyne javanica e Heterodera glycines em soja por adição de lodo de esgoto ao solo. Ciência Rural, 35:806-812.

Boeira RC, Ligo MAV \& Dynia JF (2002) Mineralização de nitrogênio em solo tropical tratado como lodos de esgoto. Pesquisa Agropecuária Brasileira, 37:1639-1647.

Cetesb (1999) Aplicação de lodos de curtume em áreas agrícolas Critérios para projeto e operação. São Paulo, Cetesb. 32p. (Manual Técnico, Norma P.4.233).

Chen J, Abawi GS \& Zucherman BM (2000) Efficacy of Bacillus thuringiensis, Paecilomyces marquandii and Streptomyces costaricanus with organic amendment against Meloidogyne hapla infecting lettuce. Journal of Nematology, 32:70-77.

Embrapa (1999) Sistema brasileiro de classificação de solos. Rio de Janeiro, Embrapa. 412p.

Ferreira, A. S.; Camargo, F. A. O.; Tedesco, M. J. \& Bissani, C (2003). A. Alterações de atributos químicos e biológicos de solo e rendimento de milho e soja pela utilização de resíduos de curtume e carbonífero. Revista Brasileira de Ciência do Solo, 27: 755-763.

Ferreira EM \& Castro IV (1995) Nodulation and growth of subterranean clover (Trifolium subterraneum L.) in soils previously treated with sewage sludge. Soil Biology and Biochemistry, 27:1171-1183.

Hussey RS \& Barker KR (1973) A comparison of methods of collecting inoculo for Meloidogyne spp. including a new technique. Plant Disease Reporter, 57:1025-1028.
Kerry BR (1987) Biological control. In: Brown, RH \& Kerry BR Principles and practice of nematode control in crops. Burnley, Academic. p. 233-262.

Lordello LGE (1988) Nematóides das plantas cultivadas. São Paulo, Nobel. 314p.

Malavolta E, Vitti GC \& Oliveira AS (1997) Avaliação do estado nutricional das plantas. Piracicaba, Associação Brasileira para pesquisa de potassa e do fosfato, 201p.

Pereira JCR, Zambolim L, Vale FXR \& Chaves GM (1996) Compostos orgânicos no controle de doenças de plantas. Revisão Anual em Proteção de Plantas, 4:353-379.

Rodriguez-Kabana R (1986) Organic and inorganic amendments to soil as nematode supressants. Journal of Nematology, 18:129135.

Soares RM, Rubin SAL, Wielewicki AP \& Ozelame JG (2004) Fungicidas no controle da ferrugem asiática (Phakospsora pachyrhizi) e produtividade da soja. Ciência Rural, 34:12451247.

Van Raij, B \& Quaggio, JA (1983) Método de análise de solo para fins de fertilidade. Campinas, IAC, Boletim Técnico, 81. 31p.

Viaene NM \& Abawi GS (1998) Fungi parasitic on juveniles and egg masses of Meloidogyne hapla in organic soils from New York. Journal of Nematology, 30:632-638.

Vieira RF (2001) Sewage sludge effects on soybean growth and nitrogen fixation. Biology and Fertility of Soils, 34:196-200.

Rev. Ceres, Viçosa, v. 57, n.5, p. 629-632, set/out, 2010 